\title{
Metamodelo de Categorização de Estilos de Aprendizagem
}

\author{
Zenaide Carvalho da Silva ${ }^{1}$, Andrey Ricardo Pimentel ${ }^{1}$ \\ ${ }^{1}$ Programa de Pós-Graduação em Informática - Universidade Federal do Paraná (UFPR) \\ Caixa Postal 19.081 - 81.531-980 - Curitiba - PR - Brasil \\ \{zcsilva, andrey\}@inf.ufpr.br
}

\begin{abstract}
Learning styles refer to preferences regarding ways and forms that the student prefers to learn. The knowledge of these styles allows propositions for teaching rearranging instructional methods and learning strategies to enable more adapted to individual student learning needs. In this context, this article presents a metamodel categorization of learning styles to help with the mapping of the preferences and characteristics of the styles that can be used to provide adaptation to the presentation of the learning object (LO) according to the student's learning style.
\end{abstract}

Resumo. Os estilos de aprendizagem referem-se a preferências em relação aos modos e formas que o aluno prefere aprender. O conhecimento desses estilos permite fazer proposições para o ensino rearranjando os métodos instrucionais $e$ as estratégias de aprendizagem para possibilitar uma aprendizagem mais adaptada as necessidades individuais do aluno. Neste contexto, este artigo apresenta um metamodelo de categorização de estilos de aprendizagem para ajudar no mapeamento das preferências e características dos estilos que podem ser usadas para proporcionar adaptação na apresentação do objeto de aprendizagem (OA) de acordo com o estilo de aprendizagem do aluno.

\section{Introdução}

Os estilos de aprendizagem (EA) referem-se a preferências individualizadas de uma pessoa, em relação aos modos e formas que ela prefere aprender no processo de ensino e aprendizagem. Cada aluno tem sua maneira de receber e processar as informações, conhecer os estilos de aprendizagem é importante para compreender melhor suas preferências individuais, e entender porque certos métodos e técnicas de ensino funcionam melhor em alguns alunos, enquanto que em outros não funcionam.

A identificação do estilo de aprendizagem é uma das maneiras para se obter a preferência e assim propiciar materiais de aprendizagem mais adequados para a aprendizagem [Valaski, Malucelli e Reinerhr 2011]. Assim destacamos a importância de considerar as preferências individuais de aprendizagem no processo de construção dos materiais de aprendizagem adaptados ao estilo do aluno, para tentar obter resultados melhores no processo de ensino e aprendizagem.

Os modelos de estilos de aprendizagem classificam os alunos quanto à sua forma ou maneira que eles preferem perceber e processar as informações recebidas quando estão aprendendo, assim suas preferências individuais de aprendizagem podem ser identificadas. Existem diversos modelos disponíveis na literatura que descrevem como 
classificar um aluno a um estilo de aprendizagem como em [Felder and Silverman 1988, Kolb 1984, Honey and Munford 2000, Butler 2003, Fleming 2001].

Cofield et al. (2004) realizaram uma investigação dos estudos sobre estilos de aprendizagem ao longo dos últimos trinta anos até 2003, identificaram setenta e um modelos de estilos de aprendizagem, categorizaram treze destes como principais modelos, considerando três critérios: sua importância teórica, sua utilização generalizada, e sua influência sobre outros modelos de estilo de aprendizagem. Apesar de observar que muitos desses modelos são pequenas adaptações de outros, ainda assim é considerado um número grande de modelos existentes na literatura.

Os modelos de estilo de aprendizagem apresentam uma série de especificidades que precisam ser observadas quando escolhemos utilizar o estilo de aprendizagem como critério de adaptação do material de aprendizagem de acordo com esses estilos. As preferências e características de cada estilo precisam ser identificadas e mapeadas para características de apresentação do material, em relação a forma e/ou formato do material a ser apresentado, assim como geração, apresentação e sequenciamento de conteúdo, ou seja, definir as características de apresentação para esse material de acordo com cada estilo do modelo a ser seguido.

Nesta perspectiva, este artigo apresenta um metamodelo de categorização de estilos de aprendizagem para ajudar no mapeamento das preferências e características dos estilos que podem ser usadas para proporcionar adaptação na apresentação do material de aprendizagem, nesse caso o objeto de aprendizagem (OA), de acordo com o estilo de aprendizagem do aluno.

O texto esta estruturado da seguinte forma. A seção 2 apresenta uma revisão sobre estilos de aprendizagem. A seção 3 apresenta os trabalhos relacionados. A seção 4 descreve aspectos metodológicos. A seção 5 apresenta o metamodelo e suas definições. A seção 6 discute aspectos do metamodelo sobre adaptação de material de aprendizagem; e, por fim, na Seção 7, são feitas as considerações finais e sugeridos trabalhos futuros.

\section{Estilos de Aprendizagem (EA)}

Estilo é a forma consistente e pessoal através da qual as pessoas usam suas qualidades e habilidades naturais para definir e conhecer a si mesmas, para experimentar o mundo imediato e relacionar-se com ele, para encontrar, avaliar, processar, criar e produzir informações [Butler 2003]. Essa definição leva em consideração algumas dimensões dos estilos: a psicológica, a afetiva e a cognitiva. No âmbito desse trabalho consideramos a dimensão cognitiva, assim se o processo de aprendizagem for adequado ao estilo, a pessoa provavelmente será mais bem sucedida como aprendiz.

A definição mais comumente aceita é de Keefe (1979), na qual o estilo de aprendizagem é uma composição de características cognitivas, afetivas e fatores fisiológicos que servem como indicadores relativamente estáveis de como um aluno percebe, interage e responde ao ambiente de aprendizagem.

O estilo de aprendizagem considerado como critério para adaptação de conteúdo em sistemas voltados para educação é um dos temas que estão cada vez mais presentes em pesquisa nacionais no âmbito educacional [Aguiar, Fechini e Costa 2014]. 
Uma investigação elaborada por Haider, Sinha e Chaudharyal (2010), mostra que as estratégias pedagógicas relacionadas ao estilo de aprendizagem do aluno contribuem para que a aprendizagem seja mais fácil, e também apresenta melhorias do processo de aprendizagem, se o material educacional usado pelo aluno estiver de acordo com seu estilo de aprendizagem. Cada estilo de aprendizagem contem características específicas que precisam ser levantadas e mapeadas, exigindo uma adaptação dos conteúdos do material educacional, no caso dessa pesquisa, vamos considerar como material educacional o objeto de aprendizagem (OA), para que o aprendiz tenha maior proveito do recurso que está sendo disponibilizado.

Existem vários modelos de estilos de aprendizagem, que foram elaborados por diversos autores, que podem ser usados pelos sistemas educacionais para representar os estilos dos alunos [Felder and Silverman 1988, Kolb 1984, Honey and Munford 2000, Butler 2003, Fleming 2001]. Os estilos de aprendizagem são definidos por esses autores de forma distintas, influenciados por diferentes teorias da psicologia da aprendizagem.

\section{Trabalhos Relacionados}

Estilos de aprendizagem incorporam as características individuais de um aluno referentes às tarefas de organizar, perceber, processar, lembrar e pensar para resolver um problema [Zaine, Bressan e Cardieri 2012]. Considerar os estilos de aprendizagem dos alunos na preparação e elaboração de material educacional possibilita atender as necessidades individuais em relação ao modo e forma que ele prefere aprender, contribuindo para um aprendizado mais adequado as suas preferências individuais de aprendizagem.

O trabalho de Radwan (2014) apresenta um sistema e-learning de curso adaptativo para apresentar o material de aprendizagem de maneiras diferentes de acordo com estilos de aprendizagem do aluno. Ele usa um sistema de gerenciamento de atividade de aprendizagem para apresentar o material, que inclui uma introdução, conteúdo, tarefas e exercícios. O sistema identifica o estilo de aprendizagem através dos questionários preenchidos pelos alunos, os resultados são usados para inferir as preferências e alterar a apresentação do material de acordo com cada estilo.

Yang, Hwang e Yang (2013) desenvolveram um sistema de aprendizagem adaptativa considerando várias dimensões de características personalizadas, propôs um módulo de apresentação personalizado para o desenvolvimento de sistemas de aprendizagem adaptativa com base no modelo de estilo cognitivo campo dependente/independente e os estilos do modelo de estilo de aprendizagem de FelderSilverman. Seus resultados experimentais mostraram que a abordagem proposta é capaz de ajudar os alunos a melhorar o seu desempenho no processo de aprendizagem.

O trabalho de Fasihuddin, Skinner e Athauda (2014) apresentou uma proposta de um modelo adaptável para personalizar os ambientes de aprendizagem abertos com base no modelo de estilos de aprendizagem de Felder-Silverman. Este modelo consiste de dois agentes principais para executar suas funcionalidades, o agente de identificação é responsável pela identificação dos estilos de aprendizagem dos alunos, monitorando certos padrões de comportamento dos alunos com os objetos de aprendizagem, enquanto o aluno interage com materiais de aprendizagem; e o agente de recomendação é 
responsável por fornecer um apoio de navegação adaptável com base nos estilos de aprendizagem e preferências identificadas.

Os trabalhos apresentados nessa seção utilizam os estilos de aprendizagem para adaptar e/ou personalizar os ambientes de aprendizagem, ou adaptar a apresentação do material de aprendizagem. A partir do metamodelo definido pretende-se realizar adaptação da apresentação do material de aprendizagem classificando as várias características e preferências dos estilos dos modelos e deduzindo características de apresentação para objetos de aprendizagem em relação a sequenciamento, apresentação e geração de conteúdo, forma/formato de avaliação e tipo e formato de recurso.

\section{Metodologia}

Existem diversos modelos de estilos de aprendizagem, elaborados por diferentes autores, que podem ser usados na identificação do perfil do aluno, alguns são mais utilizados do que outros, em virtude de ser mais indicado para ser utilizado em ambientes voltados para a educação, e/ou adequação melhor de suas escalas às características dos materiais de aprendizagem, bem como a validade e confiabilidade de seus instrumentos de medidas [Graf and Kinshuk 2007, Viola, Graf and Leo 2006, Litzinger et al. 2007]. No entanto percebe-se que grande parte deles são pequenas adaptações dos principais modelos.

Uma revisão da literatura foi realizada através da busca e investigação dos principais modelos e de maior relevância, de acordo com alguns estudos pesquisados [Akbulut and Cardak 2012, Al-Azawer and Badii 2014, Cassidy 2004, Cofield et al. 2004, Deborah, Baskaran and Kannan 2012, Feldman, Monteserin and Amandi 2014, Velusamy and Anouncias 2013].

A partir desse estudo foi identificado que muitos modelos apresentam dimensões, características e preferências semelhantes que caracterizam os estilos. Mas basicamente eles usam as três formas perceptivas de aprendizagem, a visual, a sinestésica e auditiva; alguns modelos usam todas essas três formas enquanto outros usam uma ou outra forma. Também existem outros modelos que consideram outros fatores na classificação do estilo de aprendizagem, como o modelo de Dunn e Dunn (2000) que considera os estímulos ambientais, como características que podem afetar o processo de aprendizagem.

O resultado dessa investigação foi a definição de um metamodelo de categorização de estilos de aprendizagem para ajudar no mapeamento das preferências e características dos estilos que podem ser usadas para proporcionar adaptação da apresentação do objeto de aprendizagem (OA) de acordo com o estilo de aprendizagem do aluno.

\section{Metamodelo para Categorização do Estilo de Aprendizagem (EA)}

A partir da realização do estudo e levantamento das características principais dos modelos foi definido um metamodelo para categorizar os estilos de aprendizagem através de uma representação que contemplasse uma tradução de um conjunto mínimo de elementos necessários definidos nos diversos modelos existentes na literatura. 
Através do metamodelo é possível ter uma visão genérica de todos os elementos que compõem os modelos de estilos de aprendizagem, além de permitir especificar quais elementos importantes e relevantes (no caso preferência e característica) devem ser considerados para composição de objetos de aprendizagem (OA) que possam ser adaptados de acordo com o estilo de aprendizagem do aluno. A representação desse metamodelo é visualizada na Figura 1.

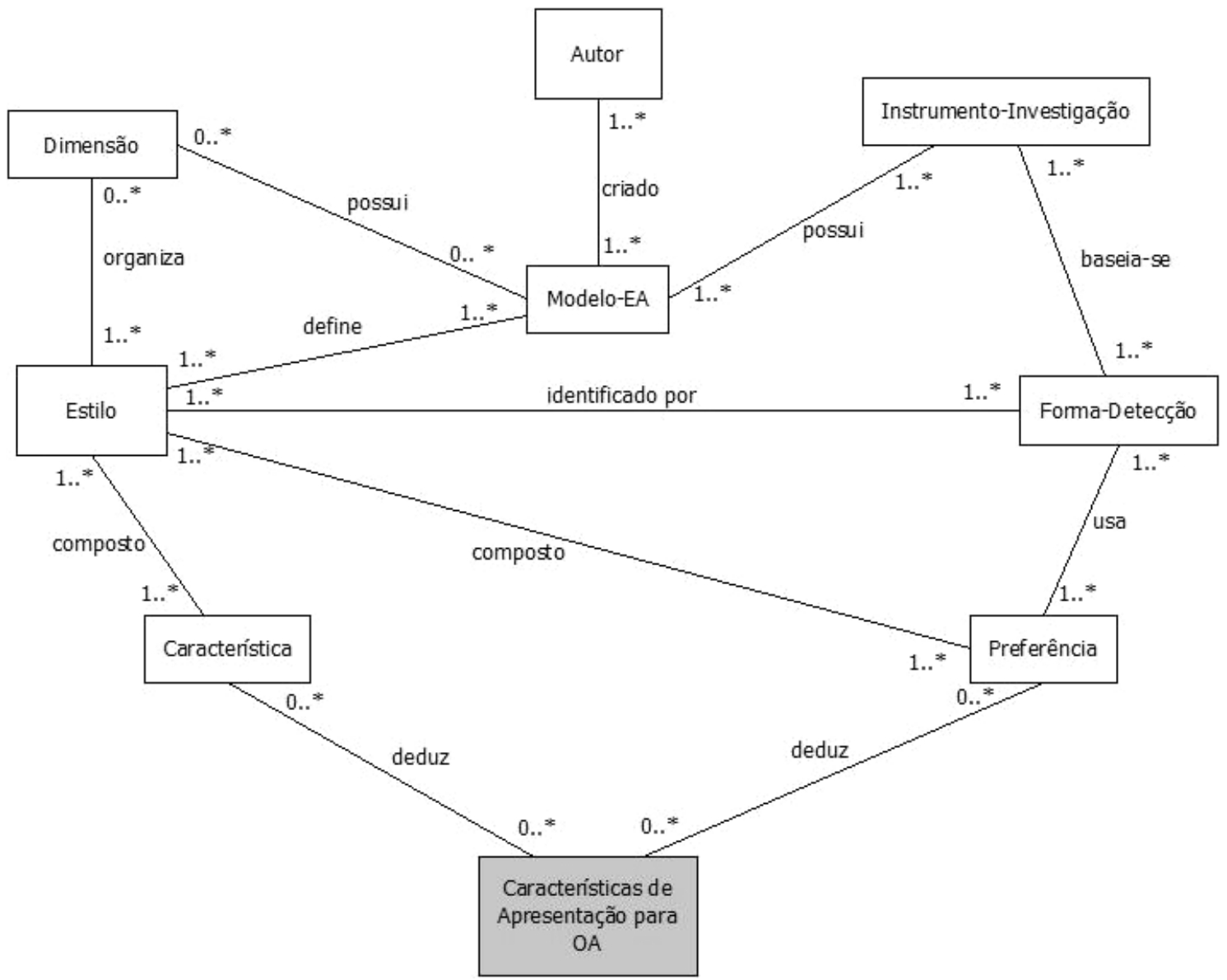

Figura 1. Metamodelo de Categorização dos Estilos de Aprendizagem (EA)

A especificação dos elementos do metamodelo priorizou a escolha de itens categorizáveis que pudesse considerar os aspectos importantes existentes na definição dos modelos de estilo de aprendizagem, além de possibilitar que a partir dos elementos preferência e característica do estilo, sejam deduzidas características de apresentação para $O A$ de acordo com cada estilo contribuindo para criação de objetos de aprendizagem adaptados ao estilo do aluno. Logo abaixo uma descrição dos elementos do metamodelo é apresentada na Tabela1, seguida de uma explanação da relação entre os elementos do metamodelo.

Tabela 1. Descrição dos elementos do metamodelo

\begin{tabular}{|l|l|}
\hline \multicolumn{1}{|c|}{ Elemento } & \multicolumn{1}{c|}{ Descrição } \\
\hline Modelo-EA & Identificação ou nome do modelo de estilo de aprendizagem. \\
\hline Autor & Nome do(s) autor (es) que criaram o modelo. \\
\hline
\end{tabular}


CBIE-LACLO 2015

Anais do XXVI Simpósio Brasileiro de Informática na Educação (SBIE 2015)

\begin{tabular}{|l|l|}
\hline Dimensão & Define a organização das categorias dos estilos do modelo. \\
\hline $\begin{array}{l}\text { Instrumento- } \\
\text { Investigação }\end{array}$ & $\begin{array}{l}\text { Mecanismo de coleta/consulta de dados composto normalmente } \\
\text { por questionários e testes psicométricos, utilizado para } \\
\text { identificação dos estilos. }\end{array}$ \\
\hline Estilo & $\begin{array}{l}\text { Nome dado a um conjunto de preferências do aluno em relação } \\
\text { aos modos e formas que ele aprende. }\end{array}$ \\
\hline Característica & $\begin{array}{l}\text { Informações gerais que compõe um estilo de aprendizagem, } \\
\text { ajudando a diferenciá-lo dos demais estilos. }\end{array}$ \\
\hline $\begin{array}{l}\text { Freferência } \\
\text { Detecção }\end{array}$ & $\begin{array}{l}\text { Preferência do aluno em relação aos modos e formas que ele } \\
\text { gosta de aprender; usada para identificar o estilo de } \\
\text { aprendizagem. }\end{array}$ \\
\hline $\begin{array}{l}\text { Características } \\
\text { de Apresentação } \\
\text { para OA }\end{array}$ & $\begin{array}{l}\text { A maneira como é coletada as informações do aluno para ser } \\
\text { usada na identificação do estilo de aprendizagem. } \\
\text { do estilo para ser usada como "elementos" para adaptação do OA } \\
\text { em relação a: sequenciamento, apresentação e geração de } \\
\text { conteúdo; forma/formato de avaliação; tipo e formato de recurso. }\end{array}$ \\
\hline
\end{tabular}

Assim de acordo com o metamodelo podemos estabelecer as seguintes relações:

1. Todo modelo de estilo de aprendizagem (Modelo-EA) é criado por um ou mais autores (Autor), possui zero ou mais dimensões (Dimensão), eles são definidos por um ou mais estilos (Estilo), e também possui um ou mais instrumentos de investigação (Instrumento-Investigação).

2. A dimensão (Dimensão) organiza um ou mais estilos (Estilo), está relacionada a zero ou mais modelo de estilo de aprendizagem (Modelo-EA).

3. O estilo de aprendizagem (Estilo) é composto por uma ou mais características (Característica), e por uma ou mais preferências (Preferência), é identificado por uma ou mais formas de detecção (Forma-Detecção), é organizado em zero ou mais dimensões (Dimensão), é definido por um ou mais modelos (Modelo$E A)$.

4. A característica (Característica) deduz zero ou mais características de apresentação para objeto de aprendizagem (OA) (Característica de Apresentação para $O A$ ), e compõe um ou mais estilos de aprendizagem (Estilo).

5. A preferência (Preferência) deduz zero ou mais características de apresentação para objeto de aprendizagem (OA) (Característica de Apresentação para $O A$ ), e compõe um ou mais estilos de aprendizagem (Estilo), é usada em um ou mais formas de detecção do estilo (Forma-Detecção).

6. A forma de detecção (Forma-Detecção) usa uma ou mais preferências (Preferência) e baseia-se em um ou mais instrumentos de investigação (Instrumento-Investigação), identifica um ou mais estilos de aprendizagem (Estilo).

A categorização dos estilos facilita a identificação dos elementos dos modelos de estilos de aprendizagem que podem ser mapeados e usados para definir um modelo de associação das características dos estilos de aprendizagem com as formas mais 
adequadas de apresentação do conteúdo do objeto de aprendizagem para o estilo de aprendizagem do aluno.

\section{Resultados e Discussão}

Ao observamos o metamodelo destacamos a importância dos elementos Característica e Preferência, pois através deles podemos identificar a qual estilo aquelas preferências e características pertencem, e consequentemente deduzir as Características de Apresentação para $O A$, pois no modelo de adaptação da apresentação do material de aprendizagem vamos usar essas informações para construir a adaptação.

Como foi descrito na tabela 1 as Características de Apresentação para $O A \mathrm{em}$ relação a sequenciamento, apresentação e geração de conteúdo, forma/formato de avaliação, tipo e formato de recurso serão usadas na adaptação da apresentação do material de aprendizagem de acordo com os estilos de aprendizagem. Assim seguindo o metamodelo, quando vamos usar o estilo de aprendizagem como critério para adaptar conteúdos de objetos de aprendizagem é preciso escolher qual o modelo de estilo de aprendizagem que vamos seguir e quais os estilos desse modelo serão utilizados, e definir as Características de Apresentação para $O A$ que serão usadas no modelo de adaptação.

A partir da definição das Características de Apresentação para $O A$ essas serão associadas a aspectos de design de interface e de interação na criação de objetos de aprendizagem, para facilitar a criação do mecanismo de composição da apresentação adaptativa dos conteúdos do objeto de aprendizagem adequadas a cada estilo.

$\mathrm{O}$ estilo se difere normalmente por preferências, assim temos que identificar as preferências de cada estilo, estabelecer o que observar e/ou monitorar dessas preferências para que se consiga obter as "diferenças" que serão associados a cada estilo, e a partir disso especificar e acomodar as necessidades individuais de cada aprendiz, através da definição das Características de Apresentação para $O A$ na composição da apresentação dos objetos de aprendizagem, fornecendo um conteúdo adaptado ao seu estilo.

$\mathrm{Na}$ Tabela 2 são apresentadas as preferências dos alunos em relação ao tipo, forma e/ou formato do recurso que devem ser observadas para composição de material de aprendizagem, seguindo as Características de Apresentação para OA, adaptado a cada estilo apenas para as dimensões Organização (como a informação é entendida) e Recebimento (como a informação é apresentada) do modelo de Felder e Silverman (1988). A partir dessas preferências observadas e mapeadas pode-se estabelecer a composição da apresentação do material de aprendizagem de acordo com o tipo e formato do recurso estabelecido para cada estilo de aprendizagem.

O modelo de estilo de aprendizagem de Felder e Silverman (1988) categoriza o estilo de aprendizagem de um aluno usando escalas dentro das quatro dimensões definidas, estabelecendo as preferências do aluno em relação as formas de processamento, percepção, recebimento e organização da informação. As dimensões Processamento (como a informação é processada) e Percepção (como a informação é percebida) não foram usadas, pois as preferências dessas dimensões não serão usadas ainda na adaptação dos conteúdos, pois não influenciam na forma e/ou formato do 
conteúdo do material de aprendizagem, serão usadas posteriormente em relação a outros itens das Características de Apresentação para OA.

Tabela 2. Preferências observadas para as dimensões Organização e Recebimento da informação dos estilos do Modelo de Felder-Silverman (1988).

\begin{tabular}{|c|c|c|c|}
\hline Dimensão & Características & Estilo & $\begin{array}{c}\text { Preferências- } \\
\text { Tipo/Formato Recurso }\end{array}$ \\
\hline \multirow[t]{2}{*}{$\begin{array}{l}\text { Recebimento } \\
\text { da Informação }\end{array}$} & $\begin{array}{l}\text { Recorda mais facilmente } \\
\text { do que viu; Privilegia as } \\
\text { informações que recebem } \\
\text { por imagem, diagramas, } \\
\text { gráficos, esquemas, } \\
\text { demonstrações. }\end{array}$ & Visual & \begin{tabular}{lr} 
Animação; & \multicolumn{2}{c}{ Diagramas; } \\
Apresentação de slides \\
com & informações \\
apresentadas no formato \\
de imagens do que texto; \\
Figuras; \\
$\begin{array}{l}\text { Demonstrações; } \\
\text { Fluxogramas; }\end{array}$
\end{tabular} \\
\hline & $\begin{array}{l}\text { Tem mais facilidade com } \\
\text { as palavras, explicações } \\
\text { escritas ou orais; Privilegia } \\
\text { o que é falado, o que está } \\
\text { escrito, as fórmulas. }\end{array}$ & Verbal & $\begin{array}{l}\text { Hipertexto; Apresentação } \\
\text { de slides com informações } \\
\text { apresentadas no formato } \\
\text { de texto; Representação } \\
\text { textual escrita ou falada. }\end{array}$ \\
\hline \multirow[t]{2}{*}{$\begin{array}{l}\text { Organização } \\
\text { da Informação }\end{array}$} & $\begin{array}{l}\text { Aprende em grandes } \\
\text { saltos, assimilando o } \\
\text { material } \\
\text { aleatoriamente, sem ver as } \\
\text { conexões, para então, } \\
\text { compreender o todo. }\end{array}$ & Global & $\begin{array}{l}\text { Links de navegação livre; } \\
\text { Problemas } \\
\text { complexos } \\
\text { detalhamento; }\end{array}$ \\
\hline & $\begin{array}{l}\text { Aprende de forma linear, } \\
\text { em etapas sequenciadas; } \\
\text { segue caminhos lógicos } \\
\text { para encontrar soluções. }\end{array}$ & Sequencial & $\begin{array}{l}\text { Links de navegação de } \\
\text { forma linear; } \\
\text { Exercício com passo a } \\
\text { passo; }\end{array}$ \\
\hline
\end{tabular}

As preferências em relação ao tipo/formato do recurso identificadas e definidas a partir das Características de Apresentação para $O A$ estabelecidas no metamodelo como as mais adequadas a cada estilo de aprendizagem serão então selecionadas para serem usadas na composição da apresentação adaptativa do OA.

\section{Conclusões e Trabalhos Futuros}

Os estilos de aprendizagem referem-se a preferências em relação aos modos e formas que o aluno prefere aprender. $\mathrm{O}$ conhecimento desses estilos permite fazer proposições para o ensino rearranjando os métodos instrucionais e as estratégias de aprendizagem para possibilitar uma aprendizagem mais adaptada as necessidades individuais do aluno.

Neste contexto, este artigo apresentou um metamodelo de categorização de estilos de aprendizagem para ajudar no mapeamento das preferências e características dos estilos que podem ser usadas para proporcionar adaptação na apresentação do objeto de aprendizagem (OA) de acordo com o estilo de aprendizagem do aluno.

Os próximos passos do trabalho serão definir a estrutura de associação das Características de Apresentação para $O A$, definidas no metamodelo, com as formas 
mais adequadas de apresentação do conteúdo do OA para cada estilo de aprendizagem, definir um mecanismo de composição da apresentação adaptativa dos conteúdos do OA a partir estrutura de associação das Características de Apresentação para OA relacionados com aspectos de design de interface e interação na criação de OA, adequados a cada estilo, e finalizar o modelo de adaptação da apresentação do objeto de aprendizagem para realizar experimentos de validação do modelo.

\section{Agradecimentos}

Os autores agradecem a Coordenação de Aperfeiçoamento de Pessoal de Nível Superior (CAPES) pelo suporte parcial a esta pesquisa.

\section{Referências}

Aguiar, J. J. B., Fechini, J. M. e Costa, E. B. (2014). Estilos Cognitivos e Estilos de Aprendizagem em Informática na Educação: um mapeamento sistemático focado no SBIE, WIE e RBIE. In XXV Simpósio Brasileiro de Informática na Educação SBIE, p.441-450.

Alaya, N. B., Elferchichi, A. and Ahmed, M. B.(2007). Towards the Elaboration of a Metamodel Unifying Learning Styles. IEEE E-Learning International Conference (ELIC2007 Conference Proceedings 2007).

Al-Azawei, A. and Badii, A. (2014). State of the Art of Learning Styles-Based Adaptive Educational Hypermedia Systems (LS-BAEHSS). Int. J. Comput. Sci. Inf. Technol, 6(3), 1-19.

Akbulut, Y. and Cardak, C. S.(2012). Adaptive educational hypermedia accommodating learning styles: A content analysis of publications from 2000 to 2011, Comput. Educ., vol. 58, no. 2, pp. 835-842.

Butler, K. A. (2003). Estilos de Aprendizagem: as dimensões psicológica, afetiva e cognitiva. Traduzido por Renata Costa de Sá Bonotto e Jorge Alberto Reichert. Porto Alegre: Editora da UFRGS, 2003.

Cassidy, S. (2004). Learning styles: An overview of theories, models, and measures. Educational Psychology, 24(4), 419-444.

Coffield, F., Moseley, D., Hall, E. and Ecclestone, K. (2004). Learning styles and pedagogy in post-16 learning: A systematic and critical review. London: Learning and Skills Research Centre.

Deborah, L. J., Baskaran, R. and Kannan A.(2012). Learning styles assessment and theoretical origin in an E-Learning scenario: a survey, Artificial Intelligence Review, Springer, DOI: 10.1007/s10462-012-9344-0.

Dunn, R. (2000). Learning styles: Theory, research, and practice. National Forum of Applied Educational Research Journal, 13 (1), 3-22.

Fasihuddin, H., Skinner, G. and Athauda, R. (2014). Towards an adaptive model to personalise open learning environments using learning styles. In Information, Communication Technology and System (ICTS), 2014 International Conference on (pp. 183-188), IEEE. 
Felder, R. M. and Silverman, L. K. (1988). Learning and Teaching Styles in Engineering Education. Journal of Engineering Education, 78(7):674-681.

Feldman, J., Monteserin, A. and Amandi, A. (2014). Automatic detection of learning styles: state of the art. Artificial Intelligence Review, 1-30.

Fleming, N. D. (2001). Teaching and learning styles: VARK strategies. Christchurch, New Zealand : N.D. Fleming.

Graf, S., and Kinshuk. (2007). Providing adaptive courses in learning management systems with respect to learning styles. In G. Richards (Ed.), Proceedings of the world conference on e-learning in corporate, government, healthcare, and higher education (e-Learn) (pp. 2576-2583). Chesapeake, VA: AACE Press.

Honey, P. and Mumford, A. (2000). The Learning Styles helper's guide. Maldenhead Berks: Peter Honey Publications.

Haider, M. T. U., Sinha, A. K., and Chaudhary, B. D. (2010). An investigation of relationship between learning styles and performance of learners. Intern. Journal of Engineering Science and Technology, 2(7):2813-2819.

Kolb, D. (1984). Experiential learning: Experience as the source of learning and development. Prentice-Hall Englewood Cliffs, NJ.

Keefe, J.W. (1979). "Learning Style: An Overview" in NASSP's Student Learning Styles: Diagonosing and Prescribing Programs" (pp. 1-17), Reston, VA: National Association of Secondary Schools.

Litzinger, T. A., Lee, S. H., Wise, J. C. and Felder, R. M.(2007). “A Psychometric Study of the Index of Learning Styles@," J. Eng. Educ., vol. 96, no. 4, pp. 309-319.

Radwan, N. (2014). An Adaptive Learning Management System Based on Learner's Learning Style. In International Arab Journal of e-Technology, Vol. 3, No. 4, 228234.

Valaski, J., Malucelli, A. e Reinehr, S. (2011). Revisão dos Modelos de Estilos de Aprendizagem Aplicados à Adaptação e Personalização dos Materiais de Aprendizagem. In XXII Simpósio Brasileiro de Informática na Educação - SBIE, p. 844-847.

Velusamy, B. and Anouncias, M. (2013) "A narrative review of research on learning styles and cognitive strategies", Journal Of Theoretical \& Applied Information Technology, 52, 1, pp. 23-29, Computers \& Applied Sciences Complete.

Viola, S., Graf, S. and Leo, T. (2006). "Analysis of Felder-Silverman Index of Learning Styles by a Data-Driven Statistical Approach", Eighth IEEE Int. Symp. Multimed., pp. 959-964.

Yang, T.C., Hwang, G.J. and Yang, S. J.H. (2013). Development of an adaptive learning system with multiple perspectives based on students' learning styles and cognitive styles. Educational Technology \& Society, 16 (4), 185-200.

Zaine, L., Bressan, A.M. e Cardieri, R. J. (2012). e-LORS Uma abordagem para recomendação de objetos de aprendizagem, Revista Brasileira de Informática na Educação. v. 20, p. 4-16. 